\title{
Glycogen storage disease due to phosphorylase kinase deficiency
}

INSERM

\section{Source}

INSERM. (1999). Orphanet: an online rare disease and orphan drug data base. Glycogen storage disease due to phosphorylase kinase deficiency. ORPHA:370

Glycogen storage disease (GSD) due to phosphorylase kinase deficiency is a group of inborn errors of glycogen metabolism that is clinically and genetically heterogeneous. This group comprises GSD due to liver phosphorylase kinase (PhK) deficiency, GSD due to muscle PhK deficiency and GSD due to liver and muscle PhK deficiency (see these terms). 